\title{
Predictive Potential of FDG-PET/CT for Histological Transformation in Patients with Indolent Lymphoma
}

\author{
Peter E. Blase ${ }^{1}$, Pieternel C. M. Pasker-de Jong ${ }^{2}$, Anton Hagenbeek ${ }^{3}$, Rob Fijnheer ${ }^{4}$, \\ Marie J. de Haas ${ }^{1}$, John M. H. de Klerk ${ }^{1}$ \\ ${ }^{1}$ Department of Nuclear medicine, Meander Medical Centre, Amersfoort, The Netherlands \\ ${ }^{2}$ Department of Meander Academy, Meander Medical Centre, Amersfoort, The Netherlands \\ ${ }^{3}$ Department of Haematology, Academical Medical Centre, Amsterdam, The Netherlands \\ ${ }^{4}$ Department of Internal medicine, Meander Medical Centre, Amersfoort, The Netherlands \\ Email: peterblase@gmail.com
}

Received November 18, 2013; revised December 18, 2013; accepted December 25, 2013

Copyright (C) 2014 Peter E. Blase et al. This is an open access article distributed under the Creative Commons Attribution License, which permits unrestricted use, distribution, and reproduction in any medium, provided the original work is properly cited. In accordance of the Creative Commons Attribution License all Copyrights (C) 2014 are reserved for SCIRP and the owner of the intellectual property Peter E. Blase et al. All Copyright (C) 2014 are guarded by law and by SCIRP as a guardian.

\begin{abstract}
Backgrounds and Purpose - In indolent non-Hodgkin lymphoma, histological transformation is a dramatic event which reduces the prognosis significantly. SUVmax values from FDG-PET/CT help differentiate between aggressive and indolent lymphomas, and transformed indolent lymphomas also show an increased FDG uptake. Possibly FDG uptake increases early in the clinical course and could predict histological transformation. Our objective was to predict histological transformation in indolent lymphomas from initial staging FDG-PET/CT. Patients and Methods-A retrospective study was performed. Patients with biopsy-proven indolent lymphoma who had had initial staging FDG-PET/CT were included. Qualitative (foci compared with FDG uptake liver) and semiquantitative (SUVmax-value per focus) analyses were performed of all abnormal foci. Patient characteristics and outcome were evaluated. Results-We included 88 patients, 5 of whom developed a histological transformation. Semiquantitative analysis showed a relation between maximum standardized uptake value and histological transformation (odds ratio 1.25, 95\% CI 1.024 - 1.513). Qualitative analysis showed a negative predictive relation of FDG uptake less than or equal to liver in the occurrence of histological transformation. Transformation-free survival was $\mathbf{1 0 0} \%$ over 30 months in those with FDG uptake lower than or equal to liver. More FDG uptake than liver showed transformation-free survival of $\mathbf{8 8 \%}$ over 30 months. Conclusion-Qualitative analysis of staging FDG-PET/CT in indolent lymphomas could be useful to rule out transformation in the next 30 months. In our study, semiquantitative analysis was statistically significantly associated with histological transformation and maximum standardized uptake value. However, because of the small number of patients, cautious interpretation of the results is warranted. More studies are needed to investigate the role of staging PET/CT in patient with indolent non-Hodkin lymphoma in the prediction of transformation.
\end{abstract}

\section{KEYWORDS}

Indolent; Lymphoma; Transformation; PET/CT; Predictive Value

\section{Introduction}

The clinical course of indolent non-Hodgkin lymphoma (NHL) is slow with a relatively long survival. Indolent lymphomas are characterized by years without symptomatology and an occasional show of progression [1-3]. Follicular lymphoma grade I-II (FL I-II) is the most common type and accounts for $70 \%$ of indolent lymphomas
[4,5]. In Europe, the incidence of follicular lymphoma is 2.18 per 100.000 per year [6]. Other frequent indolent lymphomas are chronic lymphocytic leukemia or small lymphocytic lymphoma (CLL/SLL), lymphoplasmacytic lymphoma (LPL), nodal marginal zone lymphoma (MZL), splenic marginal zone lymphoma (SMZL) and mucosa-associated lymphoid tissue lymphoma (MALT lymphoma) [5]. 
Indolent lymphomas have a remarkable clinical course and have an unusual treatment. Limited disease is treated by local involved radiotherapy. Extended disease is being treated by the principium of "wait-and-see". There is no difference in survival between the strategies "wait-andsee” and immediate start of chemotherapy [7]. Chemotherapy starts if there are mechanic or cosmetic complaints caused by enlarged lymph nodes jeopardizing function of vital organs, impending pancytopenia (due to bone marrow infiltration) and B-symptoms. Although indolent lymphomas know a relatively long survival, histological transformation reduces the prognosis dramatically. The indolent lymphoma transforms into a lymphoma with an aggressive clinical manifestation. [1,5, 8-10]. At diagnosis, 10-year survival of indolent lymphoma is $75 \%$ in non-transformed follicular lymphoma, but if follicular lymphoma undergoes histological transformation, 10-year survival from initial diagnosis is only $36 \%$ [8]. Histological transformation occurs in the first 5 years after diagnosis in $20 \%$ of follicular lymphoma cases, while $30 \%$ of the cases have transformed after 10 years $[8,11]$.

Other indolent lymphomas have a lower transformation incidence. For instance in CLL/SLL, transformation also known as Richter's syndrome occurs in $15 \%$ over 10 years [12]. After histological transformation, chemotherapy response is poor. Median survival in transformed indolent lymphomas is only 14 - 20 months after treatment $[8,9]$.

In the majority of the patients, histological transformation is accompanied by a change of the clinical behavior to a more aggressive disease. Rapidly growing lymphadenopathy [13], involvement of extranodal sites [13,14], B-symptoms [9] and raised serum lactate dehydrogenase level $[1,9,14]$ are thus suggestive for a histological transformation. The diagnosis should be confirmed by biopsy [11]. Detection of histological transformation is relatively simple, but treatment options are limited once histological transformation has occurred. It is not known whether earlier start therapy improves prognosis when histological transformation occurs. First predictive factors are therefore needed. Known risk factors for histological transformation are III or IV Ann Arbor stage disease, elevated LDH (>450 IU/L), Beta-2 microglobulin > 3 $\mathrm{mg} / \mathrm{L}$, serum albumin $<3.5 \mathrm{mg} / \mathrm{L}$, high Follicular Lymphoma International Prognostic Index (FLIPI) or International Prognostic Index (IPI) and no complete remission after initial chemotherapy $[1,10]$.

$\left[{ }^{18} \mathrm{~F}\right]$-fluorodeoxyglucose (FDG) positron emission tomography-computed tomography (PET-CT) is commonly performed in the management of patients with lymphomas. Recent studies showed that it is possible to distinguish between indolent and aggressive lymphomas. The semiquantitative "standardized uptake value" (SUV) is helpful to determine the clinical behavior of the lymphoma. High SUV indicates an aggressive lymphoma; low SUV indicates an indolent lymphoma [15-17]. Transformed indolent lymphomas also show high SUV comparable to aggressive lymphomas at the time of diagnosis of histological transformation [18]. At what moment metabolism in indolent lymphomas increases is not clear yet. As histological transformation is thought to occur locally [19], there may already be locally increased metabolism before histological transformation expresses clinically.

Our objective was to predict histological transformation from increased FDG uptake at initial staging FDGPET/CT.

\section{Patients and Methods}

\subsection{Patient Selection}

All FDG-PET/CT studies done between October 2007 and June 2012 in patients with indolent NHL were evaluated retrospectively. Patients were included if they met the following entry criteria: histopathologically confirmed indolent NHL and FDG-PET/CT for staging within 1 month of diagnosis. Patients with any treatment for indolent lymphoma before FDG-PET/CT were excluded. Patients with another diagnosis of cancer were also excluded. The study was approved by the local review committee.

\subsection{Clinical Staging}

In each patient at least one lymph node biopsy and bone marrow biopsy were performed, in one patient the biopsy was taken from the spleen. The histopathological biopsies were reviewed by pathologists of our institution, the recent WHO Classification for NHL was used [20,21]. We also collected lactate dehydrogenase (LDH), hemoglobin (Hb), lymphocyte and monocyte counts whenever available.

\section{3. ${ }^{18}$ F-FDG-PET/CT}

Studies were performed using the integrated 40-slice PET/CT scanner (Biograph 40 True Point PET-CT, Siemens Medical Systems). Patients fasted for six hours before injection of FDG. Plasma blood glucose had to be below $10 \mathrm{mmol} / \mathrm{L}$ prior to injection. Patients received intravenous FDG (3 MBq/kg body weight). After sixty minutes of rest, FDG-PET/CT was performed. A lowdose CT scan, which is used for attenuation correction of PET emission images as well as for anatomic localization of PET abnormalities, was acquired first. Then PET emission images were performed with a resolution of 1.5 mm per slice. Images were acquired from the level of the skull base to the inguinal region. 


\subsection{Image Analysis}

FDG-PET/CT scans were reviewed and reported by a nuclear medicine physician. The FDG-PET/Ct scans were reviewed without the knowledge of histologic transformation. Supervised by an experienced nuclear medicine physician (JMHK), a medical student (PEB) identified a "region of interest" (ROI) using the report. A ROI was defined as an abnormal focus with a diameter of at least $10 \mathrm{~mm}$ [22]. Each ROI was assessed both semiquantitatively and qualitatively.

For a semiquantitative evaluation the "standardized uptake value” (SUV) was calculated. The SUV was calculated for a spherical ROI of $10 \mathrm{~mm}$ diameter. This calculation was done using Siemens TrueD. The maximum SUV (SUVmax) and the mean SUV (SUVmean) was calculated for each ROI. If more than one ROI was identified, the highest SUVmax and SUVmean of the patient were recorded for further analysis. This format was chosen to study whether the most suspect ROI could be identified based on FDG uptake. The qualitative evaluation was done through visual assessment using a 4-point scale. The FDG hepatic uptake was used as reference [23,24]. Each ROI was assessed qualitatively. The highest visual assessment of each patient was recorded for further analysis.

\subsection{Statistical Analyses}

The "overall survival time" (OS) was defined as the time between the histopathological diagnosis to the time of death, regardless of the cause of death. The "transformation free survival time” (TFS) was defined as the time between the histopathological diagnosis to the time of histopathological confirmation of histological transformation. The "progression free survival time" (PFS) was defined as the time between the histopathological diagnosis to the time of radiological confirmation of progression. Patients were censored when they were lost of follow up. The OS, PFS and TFS were expressed in months.

A survival analysis was done using Kaplan-Meier curves. Log rank tests were done to demonstrate significant difference between the curves in Kaplan Meier analysis. To demonstrate a relationship between FDG uptake and the events histological transformation and progression a univariate logistic regression was done. To establish an independent relationship we conducted a multivariate logistic regression. Results were considered statistically significant when the P-value was lower than 0.05 . All statistical analyses were done using SPSS version 20.0.

\section{Results}

\subsection{General Results}

A total of 88 patients were included, each patient had biopsy-proven indolent NHL. Baseline characteristics are listed in Table 1. In our study, we identified the following histological subtypes: follicular lymphoma grade I-II (n = 42), chronic lymphocytic leukemia/small lymphocytic lymphoma $(\mathrm{n}=18)$, lymphoplasmacytic lymphoma $(n=7)$, nodal marginal zone lymphoma $(n=7)$, splenic marginal zone lymphoma $(\mathrm{n}=5)$, MALT lymphoma $(\mathrm{n}=$ $6)$, Hairy cell leukemia $(n=1)$ and unspecified indolent lymphoma $(\mathrm{n}=2)$. We identified five histological transformations in our study; each of these patients had biopsy-proven histological transformation to diffuse large Bcell lymphoma (Table 2).

Patients in our study population underwent various initial treatments (Table 1). Forty-six patients (52.3\%) underwent conservative treatment "Wait-and-see", Thirteen patients (13\%) underwent involved field radiotherapy and twenty-seven patients (30.7\%) were treated with chemo-immunotherapy. Pro diagnosis one patient underwent splenectomy and the diagnosis splenic marginal zone lymphoma was confirmed histopathologically. Another patient with localized gastric MALT lymphoma received helicobacter pylori eradication.

\subsection{Semiquantitative Analysis}

We evaluated SUV in each patient, the highest SUV value was taken for each patient for further analysis. A univariate logistic regression was done for the occurrence of histological transformation. The odds ratio was 1.25 (95\% CI 1.024 - 1.513) for each unit of SUVmax. For SUVmean the Odds Ratio was 1.45 (95\% CI 0.979 2.137) per unit of SUVmean. We performed a univariate logistic regression for progression in lymphoma. We found an odds ratio of 1.01 (95\% CI 0.924 - 1.111) per unit SUVmax. SUVmean showed an odds ratio of 0.99 (95\% CI 0.838 - 1.177). Multivariate logistic regression analysis of histological transformation showed an odds ratio 1.44 (95\% CI 1.073 - 1.932) per unit SUVmax when corrected for LDH.

\subsection{Qualitative Analysis}

The qualitative analysis was done using Kaplan-Meier curves. In patients who had only ROI with FDG uptake less than or equal to the liver, no histological transformation was observed. All five histological transformations occurred in patients with ROI with more FDG uptake than liver (Figure 1). To test the difference between the curves for statistical significance a Log Rank test was performed ( $p=0.053$ ). In the group with FDG uptake less than or equal to the liver, $100 \%$ was transformationfree over 30 months. In the group with more FDG uptake than the liver $88 \%$ was transformation-free over 30 months.

We studied the association of visual FDG uptake with 
Table 1. Baseline patient population: Patient characteristics at initial staging of indolent lymphoma $(\mathbf{n}=\mathbf{8 8})$.

\begin{tabular}{|c|c|c|c|c|}
\hline \multirow[t]{2}{*}{ Characteristics } & \multicolumn{2}{|c|}{$\begin{array}{l}\text { Non-transformed indolent lymphoma at the end of } \\
\text { follow up }(n=83)\end{array}$} & \multicolumn{2}{|c|}{$\begin{array}{l}\text { Transformed indolent lymphoma at the end of } \\
\text { follow up }(n=5)\end{array}$} \\
\hline & $\mathrm{n}$ & $\%$ & $\mathrm{n}$ & $\%$ \\
\hline Male sex & 50 & 60.2 & 1 & 20.0 \\
\hline \multicolumn{5}{|l|}{ Age } \\
\hline$>60$ years & 45 & 54.2 & 3 & 60.0 \\
\hline Mean (years) & $61(30-88)$ & & $60(27-84)$ & \\
\hline \multicolumn{5}{|l|}{ Histology of lymphoma } \\
\hline Follicular grade I-II & 40 & $48.2 \%$ & 2 & 40.0 \\
\hline CLL/small lymphocytic & 17 & $20.5 \%$ & 1 & 20.0 \\
\hline Lymphoplasmacytic & 6 & $7.2 \%$ & 1 & 20.0 \\
\hline Nodal marginal zone & 7 & $8.4 \%$ & 0 & 0.0 \\
\hline Splenic marginal zone & 4 & $4.8 \%$ & 1 & 20.0 \\
\hline MALT & 6 & $7.2 \%$ & 0 & 0.0 \\
\hline Hairy cell leukemia & 1 & $1.2 \%$ & 0 & 0.0 \\
\hline Indolent unspecified & 2 & $2.4 \%$ & 0 & 0.0 \\
\hline \multicolumn{5}{|l|}{ Extensiveness } \\
\hline B symptoms & 4 & $4.8 \%$ & 1 & 20.0 \\
\hline Bone marrow involvement & 34 & $41.0 \%$ & 2 & 40.0 \\
\hline $\begin{array}{c}\text { Involvement ( }>4 \text { lymph node areas } \\
\text { affected) }\end{array}$ & 49 & $59.0 \%$ & 2 & 40.0 \\
\hline Bulky mass $(\geq 6 \mathrm{~cm})$ & 4 & $4.8 \%$ & 2 & 40.0 \\
\hline Extranodal involvement & 17 & $20.5 \%$ & 0 & 0.0 \\
\hline Splenic involvement & 8 & $9.6 \%$ & 1 & 20.0 \\
\hline \multicolumn{5}{|l|}{ Laboratory } \\
\hline Mean lactate dehydrogenase (IU/L) & $361(128$ - 995) & & $647(463-995)$ & \\
\hline Lactate dehydrogenase $>450 \mathrm{IU} / \mathrm{L}$ & 17 & $20.5 \%$ & 5 & 100.0 \\
\hline Mean hemoglobine (mmol/L) & $8.3(5.0-10.3)$ & & $7.5(6.5-7.9)$ & \\
\hline Hemoglobine $<7.5 \mathrm{mmol} / \mathrm{L}$ & 25 & $30.1 \%$ & 1 & 20.0 \\
\hline \multicolumn{5}{|l|}{ Initial therapy } \\
\hline Wait-and-see & 45 & $54.2 \%$ & 1 & 20.0 \\
\hline Radiotherapy & 13 & $15.7 \%$ & 0 & 0.0 \\
\hline Chemo-immunotherapy & 23 & $27.7 \%$ & 4 & 80.0 \\
\hline Other & 2 & $2.4 \%$ & 0 & 0.0 \\
\hline Mean follow up (months) & $30.1(2-86)$ & & $46.0(14-86)$ & \\
\hline
\end{tabular}

Table 2. Characteristics of cases with histological transformation.

\begin{tabular}{|c|c|c|c|c|c|}
\hline Patient & 1 & 2 & 3 & 4 & 5 \\
\hline \multicolumn{6}{|c|}{ Characteristics at diagnosis } \\
\hline Histology & CLL & FL I-II & FL I-II & SMZL & LPL \\
\hline Age (years) & 27 & 57 & 60 & 84 & 70 \\
\hline Sex & F & $\mathrm{M}$ & $\mathrm{F}$ & $\mathrm{F}$ & $\mathrm{F}$ \\
\hline Staging & Binet B & $4 \mathrm{~A}$ & $4 \mathrm{~A}$ & $1 S$ & $4 \mathrm{~A}$ \\
\hline LDH (IU/L) & 995 & 463 & 726 & 553 & 496 \\
\hline SUVmean & 1.7 & 8.3 & 6.1 & 5.3 & 9.0 \\
\hline Visual & $3 / 3$ & 3/3 & 3/3 & $3 / 3$ & $3 / 3$ \\
\hline \multicolumn{6}{|c|}{ Characteristics at histological transformation } \\
\hline Time to transformation (months) & 30 & 7 & 8 & 12 & 8 \\
\hline Histology & DLBCL & DLBCL & DLBCL & DLBCL & DLBCL \\
\hline
\end{tabular}

Abbreviations: CLL, chronic lymphocytic leukemia/small lymphocytic lymphoma; FL I-II, follicular lymphoma grade I-II; SMZL, splenic marginal zone lymphoma; LPL, lymphoplasmacytic lymphoma; DLBCL, diffuse large B-cell lymphoma; M, male; F, female; LDH. Lactate dehydrogenase; SUVmax.maximum standardized uptake value; SUVmean, mean standardized uptake value. 


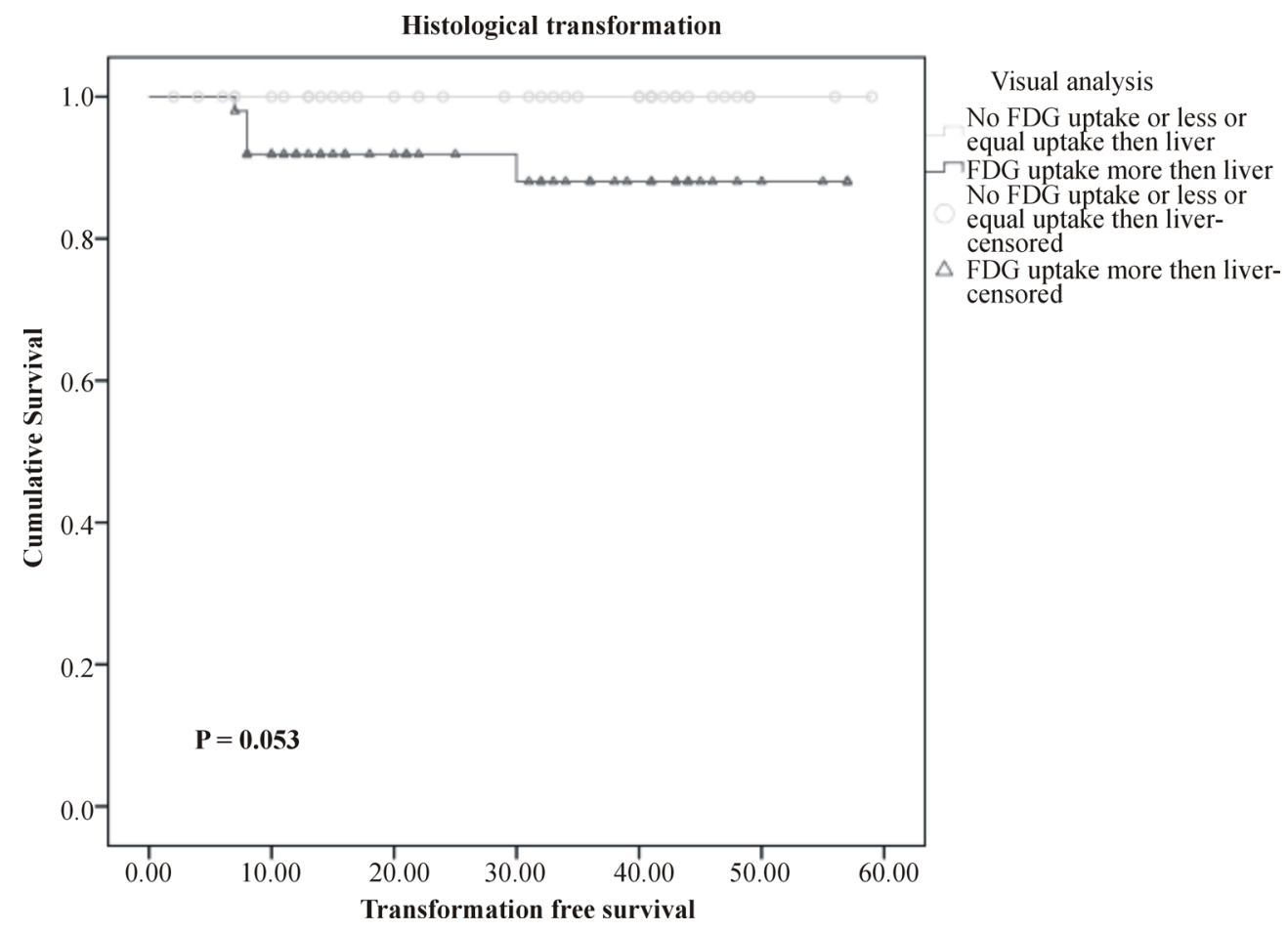

Figure 1. Kaplan-Meier survival analysis of histological transformation in indolent lymphoma. FDG uptake less or equal than liver versus FDG uptake more than liver. Transformation free survival was $\mathbf{1 0 0 \%}$ over 30 months in those with FDG uptake lower than or equal to liver. More FDG uptake than liver showed transformation free survival of $88 \%$ over 30 months. $\mathbf{P}=\mathbf{0 . 0 5 3}$.

the incidence of progression. We found no difference in the curves (p-value 0.945).

\subsection{Identification of Suspicious Foci}

We looked at each FDG-PET/CT scan of patients who underwent histological transformation in more detail. The localization where a histological transformation was diagnosed was identified on the initial FDG-PET/CT. Table 3 shows all SUVmax values of ROI in those with histological transformation. The bold values are the ROI where the histological transformation was subsequently biopsied. This table shows that in 4 of 5 cases, the maximum SUVmax value on the initial FDG-PET/CT scan was the location where the histological transformation was subsequently biopsied. The only case where biopsy was taken from the second highest SUVmax was a lymphoma with bulky mass. The highest SUVmax was also measured in this bulky intra-abdominal mass. However, this localization was difficult to biopsy because of surrounding tissue.

\subsection{Other Analyses}

We studied the other known predictors of transformation and progression. We analyzed laboratory values (LDH, $\mathrm{Hb}$, lymphocytes and monocytes) and staging parameters (Ann Arbor, bone marrow invasion, extranodal involve-
Table 3. Maximum standardized uptake value of region of interest in cases of histological transformation. Each bold SUVmax value is the localization where the histological transformation occurred.

\begin{tabular}{cccccc}
\hline ROI & Case 1 & Case 2 & Case 3 & Case 4 & Case 5 \\
\hline 1 & $\mathbf{4 . 2}(\mathbf{H T})$ & $\mathbf{1 9 . 1}(\mathbf{H T})$ & $\mathbf{1 2 . 6}(\mathbf{H T})$ & 8.5 & $\mathbf{1 1 . 4}(\mathbf{H T})$ \\
2 & 2.1 & 6.3 & 8.2 & $\mathbf{8 . 3}(\mathbf{H T})$ & 10.2 \\
3 & & 6.0 & 7.9 & 7.6 & 9.8 \\
4 & & & 7.5 & 0.9 & 9.1 \\
5 & & & 4.7 & 0.6 & 5.8 \\
6 & & & & 0.6 & 2.4 \\
\hline
\end{tabular}

Abbreviations: ROI, region of interest; HT, histological transformation.

ment, bulky mass, and number of involved lymph node areas). Univariate logistic regression showed a relationship between histological transformation and LDH, odds ratio 1.006 (95\% CI 1.003 - 1.009) per unit LDH. A multivariate logistic regression showed that LDH is an independent variable, odds ratio 1.010 per unit LDH (95\% CI 1.004 - 1.016). In our population the lowest value of LDH was $128 \mathrm{IU} / \mathrm{L}$, the highest $\mathrm{LDH}$ value was 995 IU/L, the highest LDH was the value from a patient who underwent a transformation after 30 months. We also performed a Kaplan-Meier survival analysis for increased LDH ( $\geq 450 \mathrm{IU} / \mathrm{L})$ and histological transforma- 
tion. Patients with a normal LDH $(<450 \mathrm{IU} / \mathrm{L})$ showed no histological transformation in 30 months. One third of patients with an increased LDH showed transformation in 30 months (Log rank p-value 0.124$)$. The other variables (laboratory values and staging parameters as described above) did not show any relationship.

\subsection{SUVmax by Histological Subtype}

The different SUVmax varied by histological subtype are shown in Figure 2. Follicular lymphoma, the largest group, showed a wide range, and so did MALT-lymphoma. Other groups showed a smaller range. When we compared transformed lymphoma by histological subtype (Figure 2: lines 17, 97, 475, 658 and 743) versus nontransformed lymphoma, we observed that SUVmax was at the high end of the spectrum for all transformed lymphomas.

\section{Discussion}

We observed a statistically significant association between SUVmax and histological transformation. Patients who had more FDG uptake in the ROI than the liver were at risk of histological transformation at the site of the highest SUV. In patients who had FDG-uptake less than or equal. The group with less or equal FDG uptake than liver has a small risk of transformation. This information provides important insight in the prognosis for patients with indolent lymphoma. Patients with high initial FDG uptake are at risk for transformation and negative outcome.

Visual assessment is influenced by the background of the ROI, this makes assessment less reliable. The advantage of visual assessment is that, as FDG uptake is compared with FDG uptake in the liver, this corrects the reading for background uptake.

The group with more FDG uptake in the lymph nodes than in the liver is interesting because these patients may be at risk for histological transformation. When further distinction can be made in degree of FDG uptake we might find that there is a smaller group at risk for histological transformation. For example, by comparing the ROI with the cerebellum, which has also a relatively constant glucose metabolism and which has a higher FDG uptake than the liver $[25,26]$, we might be able to identify the areas and thus persons with the highest risk of transformation.

The SUVmax in different types of NHL can vary. In three retrospective studies (Karam [27], Schöder [17] and Papajik [28]), including 131 patients, the relation between the mean SUVmax by histological subtype was determined. The results of these studies are shown in Table 4. We calculated a weighted average from these studies for the comparison with our results. In our study

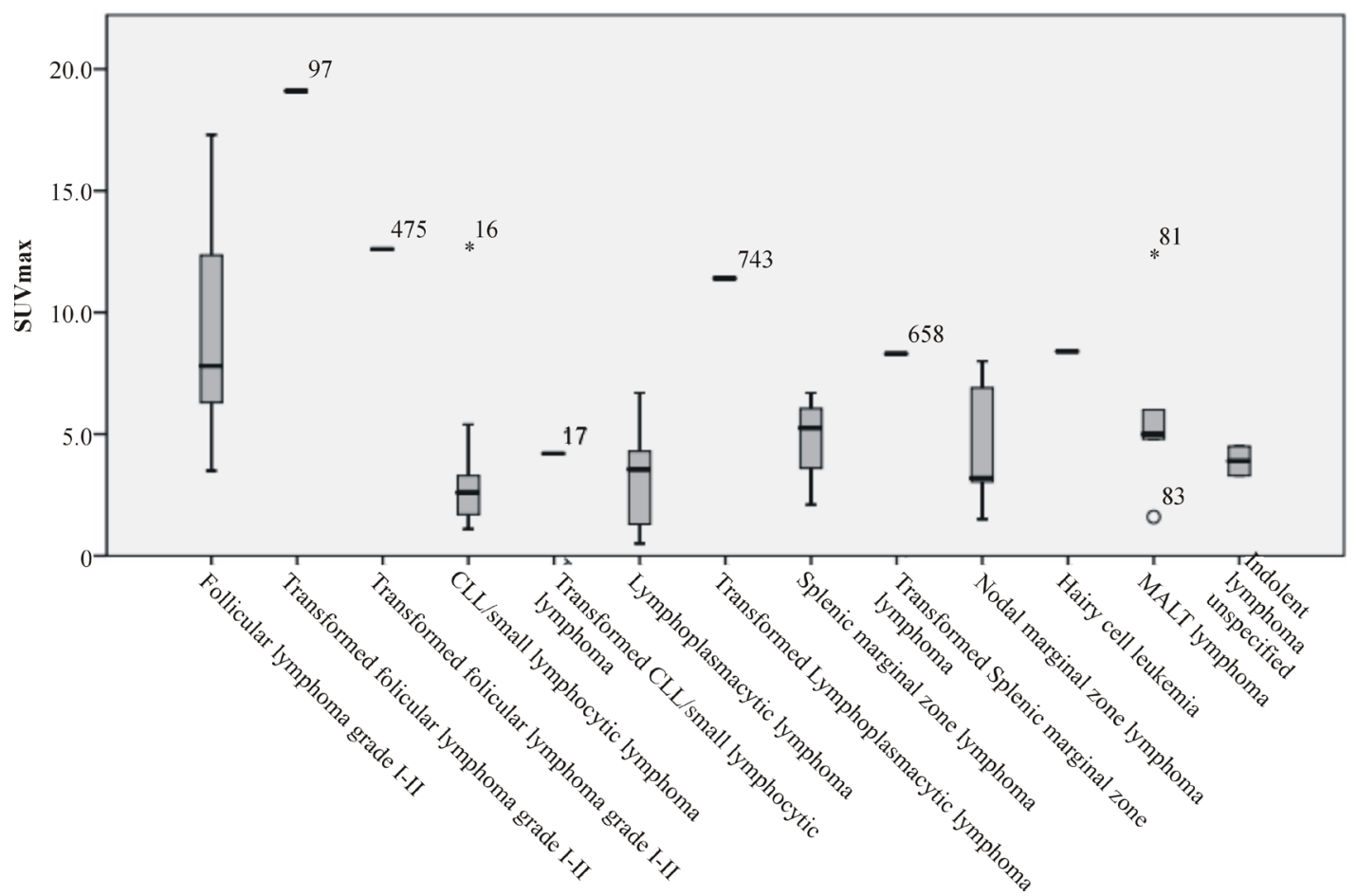

Figure 2. Box-plot of SUVmax value per histological subtype of non-transformed indolent lymphoma. Transformed indolent lymphomas were patient 17, 97, 475, 658 and 743. Outlier and extremes were patient 16, 81 and 83 . Values were compared with transformed indolent lymphoma per histological subtype. Transformed lymphomas were at the high end of the spectrum per histological subtype. 
Table 4. Maximum standardized uptake values by histological subtype.

\begin{tabular}{|c|c|c|c|c|c|c|c|c|c|c|c|c|}
\hline \multirow[b]{2}{*}{ Lymphoma } & \multicolumn{3}{|c|}{ Karam et al. [27] } & \multicolumn{3}{|c|}{ Schöder et al. [17] } & \multicolumn{3}{|c|}{ Papajik et al. [28] } & \multicolumn{3}{|c|}{ Current study } \\
\hline & $\begin{array}{c}\text { Mean } \\
\text { SUVmax }\end{array}$ & $\begin{array}{c}\text { No. of } \\
\text { patients }\end{array}$ & $S D$ & $\begin{array}{c}\text { Mean } \\
\text { SUVmax }\end{array}$ & $\begin{array}{c}\text { No. of } \\
\text { patients }\end{array}$ & $S D$ & $\begin{array}{c}\text { Mean } \\
\text { SUVmax }\end{array}$ & $\begin{array}{c}\text { No. of } \\
\text { patients }\end{array}$ & Range & $\begin{array}{c}\text { Mean } \\
\text { SUVmax }\end{array}$ & $\begin{array}{c}\text { No. of } \\
\text { patients }\end{array}$ & $S D$ \\
\hline $\begin{array}{l}\text { CLL/small } \\
\text { lymphocytic }\end{array}$ & 2.5 & 15 & 0.7 & 6.4 & 1 & & 3.7 & 7 & $2.1-5.6$ & 3.2 & 17 & 2.7 \\
\hline Lymphoplasmacytic & & & & 7.4 & 1 & & & & & 3.3 & 6 & 2.2 \\
\hline $\begin{array}{c}\text { Nodal marginal } \\
\text { zone }\end{array}$ & 3.7 & 16 & 1.4 & 8.8 & 4 & 4.9 & 4.9 & 11 & $2.0-12.5$ & 4.7 & 7 & 2.5 \\
\hline
\end{tabular}

we observed a variation per histological subtype in mean SUVmax (Figure 3), there were 3 exceptions observed. Patient number 16 had CLL/SLL and did not have pathological lymphomas, however she had sharp bone marrow invasion in the axial skeleton. Patients number 81 and 83 were two patients with MALT lymphoma. In the literature there is also a wide range of SUVmax values in MALT lymphoma, ranging from 1.4 to 26.0 [29, 30]. Follicular lymphoma grade I-II in our study also showed a wide range. This wide range has been reported before and is associated with combining the histological grade I and II [31]. We compared our results with the results of Schöder [17], Karam [27] and Papajik [28], shown in Table 4. The mean data show similarity in follicular lymphoma and CLL/SLL. The mean value in marginal zone lymphoma looks similar; however we see wide variation between the separate mean SUVmax values. Because the distribution of SUVmax values is skewed, the use of mean values is inappropriate. These data could thus only give a slight hint of interpretation of data in future research as a baseline score.

We compared transformed indolent lymphoma versus non-transformed indolent lymphoma by histological subtype. Patients who later developed transformations had SUV values that were among the highest for the histological subtype. The definition of increased FDG uptake might be different for the various histological subtypes. However, we do not now have enough cases to be able to substantiate this. A larger patient population is necessary to determine cut off values for SUVmax to identify patients at increased risk of histological transformation by histological subtype. In four of our 5 cases of histological transformation the localization of histological transformation was identifiable on the diagnostic scan as the area with the highest SUVmax. The fifth had a slightly higher uptake in a less accessible location. We do not know for sure whether transformation had occurred in that area as well.

Searches of available literature revealed that there were no studies that focused primarily on FDG uptake as a risk predictor for histological transformation. Some studies which focused on indolent lymphoma and histological transformation reported cases where FDG uptake
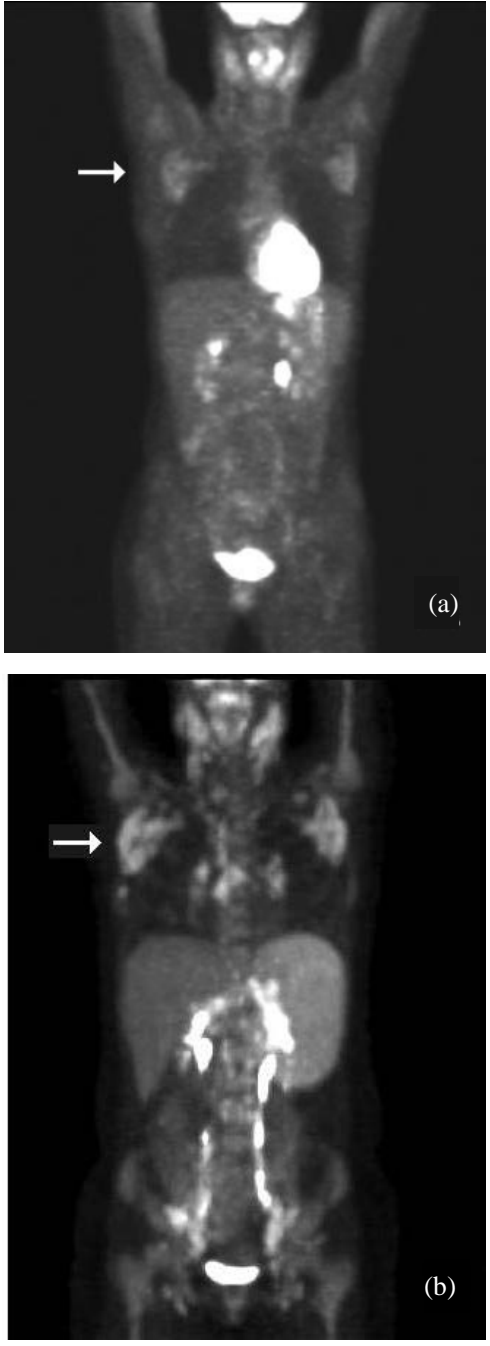

Figure 3. A patient with histolpathological diagnosis Chronic lymphocytic leukemia (CLL/SSL). (a) Axillary lymphoma SUVmax = 4.2 (baseline SUVmax for CLL/SLL $=3.0$ ). She was treated with $6 x$ fludarabine/cyclophosphamide. (b) After 25 months patient presented with weight loss, progressive lymphadenopathy and splenomegaly. Histopathological diagnosis of axillary biopsy showed histological transformation to diffuse large B-cell lymphoma. FDGPET/CT were performed and showed extended lymphadenopathy and bone marrow invasion. Axillary lymphoma showed SUVmax $=10.1$. She was treated with R-DHAP and prepared for autologous stem cell transplantation. 
was reviewed semiquantitatively $[10,19]$. However they did not present baseline scans in relation to time to histological transformation.

A retrospective study by Noy et al. examined SUVmax values in transformed indolent lymphomas [10]. Forty patients were included. The SUVmax values ranged from 3 to 38, mean 15. In this study, FDG-PET scans at time of diagnosis of indolent lymphoma were available for 12 patients. SUVmax was calculated for the area where biopsy of indolent lymphoma was performed later. Highest SUVmax elsewhere on the scan were recorded in case the indolent lymphoma had already been biopsied $(n=4)$. The SUVmax at diagnosis was not calculated for the localization of histological transformation. In all patients they observed, the SUVmax increased from indolent lymphoma to histological transformed lymphoma. In 8 of 12 patients, the SUVmax increased by more than 50\%. Mean SUVmax of patients with follicular lymphoma $(n=6)$, at time of diagnosis of indolent lymphoma was 10.8 (range 3.0 - 16.1). In marginal zone lymphoma $(\mathrm{n}=4)$ it was 5.9 (range 3.8 - 6.3). In one CLL/SLL patient SUVmax was 5.5. Compared to mean baseline SUVmax in non-transformed patients from the study by Karam, Schöder, and Papajik, the mean value of the follicular lymphomas that would later transform was 2.1 points higher than the value in non-transformed follicular lymphomas. However, two patients with a histological transformation did not have a higher SUVmax value than patients with no histological transformation. In the study of Noy et al. all indolent lymphomas which later transformed were at the high end of the spectrum for their histological subtype. However, again overlap with non-transformed lymphoma was always present [10].

A retrospective study of Karam et al. examined SUVmax values before and after histological transformation [19]. They analyzed 29 patients with histological transformation, and 41 patients with an indolent lymphoma. the indolent lymphomas were of the following types: follicular lymphoma $(\mathrm{n}=25)$, CLL/SLL $(\mathrm{n}=8)$, and marginal zone lymphoma $(n=8)$. Among those with histological transformation, 12 patients had FDG-PET/ CT before and after histological transformation. Of these, 7 had a follicular lymphoma, 3 had a CLL/SLL, and two had marginal zone lymphoma. SUVmax was calculated at the location of diagnostic biopsy in both indolent lymphoma and histological transformation. Before histological transformation mean SUVmax was 7.3 (SD 4.4). Between transformed en non-transformed indolent lymphoma was an overlap in SUVmax value. Time between both diagnoses was not reported. Data were only reported in values by group and not by histological subtype or independently. There was also a large overlap in standard deviation [19].

Our study has some limitations. Firstly, we took the highest SUV value for each patient on the diagnostic scan. If the SUV value would be determined only in the localization of the diagnostic biopsy, it would probably be lower. Furthermore, sometimes the involved lymph node is removed completely, leaving no more SUV to assess.

Secondly, the number of cases of histological transformation was low in this study. A larger study may provide more power to confirm or disprove our hypothesis that SUV-max at original staging may predict histological transformation in patients with indolent lymphoma. Available literature has not previously addressed this relation.

\section{Conclusion}

Our objective was to predict histological transformation from the degree of FDG uptake on the staging FDG-PET/ CT scan on the hypothesis that glucose uptake might be a marker for activity of the tumor.

The semiquantitative analysis showed a relation between the SUVmax and the occurrence of histological transformation. In qualitative analysis we found that histological transformation only took place in indolent lymphoma that had a higher FDG uptake than liver. So, staging FDG-PET/CT may be useful to predict the occurrence of histological transformation.

\section{Acknowledgments}

We gratefully acknowledge the suppletion of patient data by P. J. Hagen, V. Bongers (Department of Nuclear medicine, Diakonessenhuis, Utrecht, The Netherlands), R. van der Griend (Department of Internal medicine, Diakonessenhuis, Utrecht, The Netherlands), A. C. Dijk (Department of Internal medicine, Sint Jansdal, Harderwijk, The Netherlands), S. K. Klein (Department of Internal medicine, Meander Medical Centre, Amersfoort, The Netherlands).

\section{REFERENCES}

[1] Y. Bastion, C. Sebban, F. Berger, P. Felman, G. Salles, C. Dumontet, et al., "Incidence, Predictive Factors, and Outcome of Lymphoma Transformation in Follicular Lymphoma Patients," Journal of Clinical Oncology, Vol. 15, No. 4, 1997, pp. 1587-1594.

[2] S. M. Hubbard, B. A. Chabner, V. T. DeVita, R. Simon, C. W. Berard. R. B. Jones, et al., "Histologic Progression in Non-Hodgkin's Lymphoma,” Blood, Vol. 59, No. 2, 1982, pp. 258-264.

[3] D. L. Oviatt, J. B. Cousar, R. D. Collins, J. M. Flexner and R. S. Stein, "Malignant Lymphomas of Follicular Center Cell Origin in Humans. V. Incidence, Clinical Features, and Prognostic Implications of Transformation of Small Cleaved Cell Nodular Lymphoma," Cancer, Vol. 
53, No. 5, 1984, pp. 1109-1114. http://dx.doi.org/10.1002/1097-0142(19840301)53:5<110 9::AID-CNCR2820530516>3.0.CO;2-O

[4] A. G. Glass, L. H. Karnell and H. R. Menck, “The National Cancer Data Base Report on Non-Hodgkin's Lymphoma," Cancer, Vol. 80, No. 12, 1997, pp. 2311- 2320. http://dx.doi.org/10.1002/(SICI)1097-0142(19971215)80: 12<2311::AID-CNCR13>3.0.CO;2-X

[5] A. Smith, D. Howell, R. Patmore, A. Jack and E. Roman, "Incidence of Haematological Malignancy by Sub-Type: A Report from the Haematological Malignancy Research Network,” British Journal of Cancer, Vol. 105, No. 11, 2011, pp. 1684-1692.

http://dx.doi.org/10.1038/bjc.2011.450

[6] M. Sant, C. Allemani, C. Tereanu, R. De Angelis, R. Capocaccia, O. Visser, et al., "Incidence of Hematologic Malignancies in Europe by Morphologic Subtype: Results of the HAEMACARE Project," Blood, Vol. 116, No. 19, 2010, pp. 3724-3734. http://dx.doi.org/10.1182/blood-2010-05-282632

[7] K. M. Ardeshna, P. Smith, A. Norton, B. W. Hancock, P. J. Hoskin, K. A. MacLennan et al., "Long-Term Effect of a Watch and Wait Policy Versus Immediate Systemic Treatment for Asymptomatic Advanced-Stage Non-Hodgkin Lymphoma: A Randomised Controlled Trial,” Lancet, Vol. 362, No. 9383, 2003, pp. 516-522. http://dx.doi.org/10.1016/S0140-6736(03)14110-4

[8] A. J. Al-Tourah, K. K. Gill, M. Chhanabhai, P. J. Hoskins, R. J. Klasa and K. J. Savage, "Population-Based Analysis of Incidence and Outcome of Transformed Non-Hodgkin’s Lymphoma,” Journal of Clinical Oncology, Vol. 26, No. 32, 2008, pp. 5165-5169.

http://dx.doi.org/10.1200/JCO.2008.16.0283

[9] S. Montoto, A.J. Davies, J. Matthews, M. Calaminici, A. J. Norton, J. Amess, "Risk and Clinical Implications of Transformation of Follicular Lymphoma to Diffuse Large B-Cell Lymphoma," Journal of Clinical Oncology, Vol. 25, No. 17, pp. 2426-2433. http://dx.doi.org/10.1200/JCO.2006.09.3260

[10] A. Noy, H. Schöder, M. Gönen, M. Weissler, K. Ertelt and C. Cohler, "The Majority of Transformed Lymphomas Have High Standardized Uptake Values (SUVs) on Positron Emission Tomography (PET) Scanning Similar to Diffuse Large B-Cell Lymphoma (DLBCL)," Annals of Oncology, Vol. 20. No. 3, 2009, pp. 508-512. http://dx.doi.org/10.1093/annonc/mdn657

[11] S. Montoto and J. Fitzgibbon, "Transformation of Indolent B-Cell Lymphomas,” Journal of Clinical Oncology. Vol. 29, No. 14, 2011, pp. 1827-1834. http://dx.doi.org/10.1200/JCO.2010.32.7577

[12] A. Molica, “A Systematic Review on Richter Syndrome: What Is the Published Evidence?” Leuk Lymphoma, Vol. 51, No. 3, 2010, pp. 415-421. http://dx.doi.org/10.3109/10428190903515192

[13] D. Rossi, M. Cerri, D. Capello, C. Deambrogi, F. M. Rossi, A. Zucchetto, et al., "Biological and Clinical Risk Factors of Chronic Lymphocytic Leukaemia Transformation to Richter Syndrome,” British Journal of Haematology, Vol. 142, No. 2, 2008, pp. 202-215.
http://dx.doi.org/10.1111/j.1365-2141.2008.07166.X

[14] P. Lin, A. Mansoor, C. Bueso-Ramos, S. Hao, R. Lai and L. J. Medeiros, "Diffuse Large B-cell Lymphoma Occurring in Patients with Lymphoplasmacytic Lymphoma/Waldenstrom Macroglobulinemia. Clinicopathologic Features of 12 Cases," American Journal of Clinical Pathology, Vol. 120, No. 2, 2003, pp. 246-253. http://dx.doi.org/10.1309/R01VXG46MFCDVNHL

[15] M. Lapela, S. Leskinen, H. R. Minn, P. Lindholm, P. J. Klemi and K. O. Söderstrom, "Increased Glucose Metabolism in Untreated Non-Hodgkin's Lymphoma: A Study with Positron Emission Tomography and Fluorine-18Fluorodeoxyglucose,” Blood, Vol. 86, No. 9, 1995, pp. 3522-3527.

[16] M. Rodriguez, S. Rehn, H. Ahlstrom, C. Sundstrom and B. Glimelius, "Predicting Malignancy Grade with PET in Non-Hodgkin's Lymphoma,” Journal of Nuclear Medicine, Vol. 36, No. 10, 1995, pp. 1790-1796.

[17] H. Schöder, A. Noy, M. Gönen, L. Weng, D. Green and Y. E. Erdi, "Intensity of 18 Fluorodeoxyglucose Uptake in Positron Emission Tomography Distinguishes between Indolent and Aggressive Non-Hodgkin's Lymphoma," Journal of Clinical Oncology, Vol. 23, No. 21, 2005, pp. 4643-4651.http://dx.doi.org/10.1200/JCO.2005.12.072

[18] C. Bodet-Milin, F. Kraeber-Bodere, P. Moreau, L. Campion, B. Dupas and G. S. Le, "Investigation of FDGPET/CT Imaging to Guide Biopsies in the Detection of Histological Transformation of Indolent Lymphoma," Haematologica, Vol. 93, No. 3, pp. 471-472.

http://dx.doi.org/10.3324/haematol.12013

[19] M. Karam, P. J. Feustel, C. D. Vera and T. Nazeer, "Features of Large Cell Transformation of Indolent Lymphomas as Observed on Sequential PET/CT," Nuclear Medicine Communications, Vol. 32, No. 3, 2011, pp. 177-185. http://dx.doi.org/10.1097/MNM.0b013e328342b9d2

[20] N. L. Harris, E. S. Jaffe, J. Diebold, G. Flandrin, H. K. Muller-Hermelink, J. Vardiman, et al., "The World Health Organization Classification of Neoplastic Diseases of the Hematopoietic and Lymphoid Tissues. Report of the Clinical Advisory Committee Meeting, Airlie House, Virginia, November 1997," Annals of Oncology, Vol. 10, No. 12, 1999, pp. 1419-1432. http://dx.doi.org/10.1023/A:1008375931236

[21] N. L. Harris, E. S. Jaffe, J. Diebold, G. Flandrin, H. K. Muller-Hermelink, J. Vardiman, et al., "Lymphoma Classification-From Controversy to Consensus: The R.E.A. L. and WHO Classification of Lymphoid Neoplasms," Annals of Oncology, Vol. 11, 2000, Suppl. 1, pp. 3-10. http://dx.doi.org/10.1093/annonc/11.suppl_1.S3

[22] R. Boellaard, M. J. O’Doherty, W. A. Weber, F. M. Mottaghy, M. N. Lonsdale, S. G. Stroobants, et al., "FDG PET and PET/CT: EANM Procedure Guidelines for Tumour PET Imaging: Version 1.0," European Journal of Nuclear Medicine and Molecular Imaging, Vol. 37, No. 1, 2010, pp. 181-200. http://dx.doi.org/10.1007/s00259-009-1297-4

[23] S. F. Barrington, W. Qian, E. J. Somer, A. Franceschetto, B. Bagni, E. Brun, et al., "Concordance between Four European Centres of PET Reporting Criteria Designed for 
Use in Multicentre Trials in Hodgkin Lymphoma,” European Journal of Nuclear Medicine and Molecular Imaging, Vol. 37, No. 10, 2010, pp. 1824-1833. http://dx.doi.org/10.1007/s00259-010-1490-5

[24] S. F. Barrington, J. E. MacKewn, P. Schleyer, P. K. Marsden, N. G. Mikhaeel, W. Qian et al., "Establishment of a UK-Wide Network to Facilitate the Acquisition of Quality Assured FDG-PET Data for Clinical Trials in Lymphoma,” Annals of Oncology, Vol. 22, No. 3, 2011, pp. 739-745.http://dx.doi.org/10.1093/annonc/mdq428

[25] B. Tang, J. Malysz, V. Douglas-Nikitin, R. Zekman, R. H. Wong, I. Jaiyesimi, et al., "Correlating Metabolic Activity with Cellular Proliferation in Follicular Lymphomas," Molecular Imaging and Biology, Vol. 11, No. 5, 2009, pp. 296-302. http://dx.doi.org/10.1007/s11307-009-0226-z

[26] S. Zincirkeser, E. Sahin, M. Halac and S. Sager, "Standardized Uptake Values of Normal Organs on 18F-Fluorodeoxyglucose Positron Emission Tomography and Computed Tomography Imaging," Journal of International Medical Research, Vol. 35, No. 2, 2007, pp. 231236.http://dx.doi.org/10.1177/147323000703500207

[27] M. Karam, L. Novak, J. Cyriac, A. Ali, T. Nazeer, F. Nugent, "Role of Fluorine-18 Fluoro-Deoxyglucose Positron Emission Tomography Scan in the Evaluation and Follow-Up of Patients with Low-Grade Lymphomas,”
Cancer, Vol. 107, No. 1, 2006, pp. 175-183.

http://dx.doi.org/10.1002/cncr.21967

[28] T. Papajík, M. Myslivecek, Z. Sedová, E. Buriánková, V. Procházka, P. Koranda, et al., "Standardised Uptake Value of 18F-FDG on Staging PET/CT in Newly Diagnosed Patients with Different Subtypes of Non-Hodgkin's Lymphoma,” European Journal of Haematology, Vol. 86, No. 1, 2011, pp. 32-37. http://dx.doi.org/10.1111/j.1600-0609.2010.01532.x

[29] K. P. Beal, H. W. Yeung and J. Yahalom, "FDG-PET Scanning for Detection and Staging of Extranodal Marginal Zone Lymphomas of the MALT Type: A Report of 42 cAses," Annals of Oncology, Vol. 16, No. 3, 2005, pp. 473-480.http://dx.doi.org/10.1093/annonc/mdi093

[30] M. Hoffmann, S. Wöhrer, A. Becherer, A. Chott, B. Streubel, K. Kletter, et al., "18F-Fluoro-Deoxy-Glucose Positron Emission Tomography in Lymphoma of Mucosa-Associated Lymphoid Tissue: Histology Makes the Difference,” Annals of Oncology, Vol. 17, No. 12, 2006, pp. 1761-1765. http://dx.doi.org/10.1093/annonc/mdl295

[31] L. T. Tan and K. L. Ong, "Semi-Quantitative Measurements of Normal Organs with Variable Metabolic Activity on FDG PET Imaging," ANNALS Academy of Medicine Singapore, Vol. 33, No. 2, 2004, pp. 183-185. 